\title{
Les Déterminants Idiosyncratiques De La Performance Bancaire Au Maroc : Analyse Sur Données De Panel
}

\author{
Salwa Bahyaoui, (Enseignante-chercheur) \\ Faculté des Sciences Juridiques, Economiques et Sociales - Agdal- \\ Université Mohammed V de Rabat, Maroc
}

doi: 10.19044/esj.2017.v13n13p57 URL:http://dx.doi.org/10.19044/esj.2017.v13n13p57

\begin{abstract}
If the financial crisis of 2008 raised the interest in analyzing the impact of the financial system, particularly the banking one, on the real economy ; studies were focused on macroeconomic variables given the crisis fallout. However, in spite of its propagation to the international scale, the financial crisis has impacted more severely some developed economies. Rightly, the Moroccan banking system was slightly affected. This suggests a specificity of the Moroccan context. The aim of this study is, therefore, to apprehend the banking performance in Morocco from a microeconomic point of view. To do so, a panel data analysis relative to eight banks was conducted over the period 2004-2015. The results allowed to identify the determinants of banking performance in the Moroccan context while confirming certain specificities of the banking system in Morocco.
\end{abstract}

Keywords: Performance, Banks, Panel data, Morocco, Determinants

\section{Résumé}

Si la crise financière de 2008 a ressuscité l'intérêt de l'analyse de l'impact du système financier en général et du système bancaire en particulier sur l'économie réelle, les études se sont focalisées sur les grandeurs macroéconomiques au vu des retombées de ladite crise. Toutefois, et en dépit de sa propagation à l'échelle internationale, elle a touché plus sévèrement certaines économies notamment développées. A juste titre, le système bancaire marocain a été légèrement affecté. Ce qui laisse présager une spécificité du contexte marocain. L'objet de cette étude est donc d'appréhender la performance bancaire au Maroc d'un point de vue microéconomique. Pour ce faire, une analyse sur données de panel relatives à huit banques a été menée sur la période 2004-2015. Les résultats ont permis d'identifier les déterminants de la performance bancaire dans le contexte 
marocain, tout en confortant certaines spécificités du système bancaire au Maroc.

Mots-clés: Performance, Banques, Données de panel, Maroc, Déterminants.

\section{Introduction}

La contribution du secteur bancaire au financement de l'économie, à travers sa fonction d'intermédiation financière, a depuis longtemps suscité l'intérêt de plusieurs chercheurs (King \& Levine, 1993 ; Easterly, 1993 ; Levine, 1997, 2004 ; Deisting et al., 2012). En outre, le consensus est presque général quant à l’impact positif du développement financier sur la croissance économique (Tsafack Nanfosso \& Nguena, 2015).

Or, pour assurer la pérennité de cette fonction, les banques doivent consolider leur performance. Cette consolidation est doublement importante. D’abord, pour la banque, en tant qu'organisation à but lucratif, ayant des comptes à rendre à ses propres actionnaires; et donc, à ce titre doit leur assurer une rentabilité satisfaisante. Ensuite, vis-à-vis du tissu économique dans son ensemble, car la performance bancaire a des implications fortes sur la croissance économique.

En effet, la succession des crises financières ayant touché les systèmes bancaires à l'échelle mondiale, a (dé)montré leur impact fortement négatif sur le reste de l'économie. Aglietta \& Brand (2015), en référence à Reinhart \& Rogoff (2009), admettent que « les crises financières de grande ampleur provoquent une dépression dans la production, l'emploi et l'accumulation du capital ». En rapprochant les trois crises majeures, à savoir la grande déflation de 1873, la dépression des années 1930 et la crise récente de 2008, ils considèrent que « L’événement inaugural commun dans les trois cas reste une crise financière ».

En outre, l’ouverture des économies accentue le phénomène en accélérant la propagation de la crise au-delà des frontières où elle a pris naissance. La crise financière de 2008 et l'ampleur des dégâts qu'elle a entrainé au niveau de l'économie réelle dans plusieurs pays, témoignent de la forte imbrication des sphères financière et réelle.

Dans ce contexte, tous les pays touchés par la crise, notamment ceux développés, se sont concertés pour en trouver une issue (OCDE, 2009). Les analyses se sont, alors, focalisées sur le risque systémique et des «stress tests » sont, dorénavant, menés pour évaluer les risques de contagion en cas de nouvelle crise. Mais au-delà des solutions apportées, deux questions majeures se sont posées avec acuité :

Pourquoi, en dépit des règles prudentielles mises en place et régulièrement révisées par les banques centrales, la crise n’a pas pu être prévue, encore moins évitée? 
- $\quad$ Quelles mesures faut-il désormais prendre pour prévenir la survenue de telles crises dans le futur?

Plusieurs études ont tenté de répondre à ces questions, se plaçant le plus souvent d'un point de vue macroéconomique (Stiglitz, 2010a, 2010b). Par ailleurs, même si la crise s'est mondialisée, son impact ne s'est pas ressenti de la même manière dans tous les pays. C'est le cas, entre autres, des banques marocaines qui se trouvent épargnées, malgré une légère régression de leurs indicateurs de performance durant la période de la crise. Ce qui laisse présager une spécificité du contexte marocain.

Ainsi, l’objectif principal de cet article est d'appréhender la performance des banques marocaines d'un point de vue micro-économique, et ce en tentant d'identifier les principaux déterminants idiosyncratiques de ladite performance. Cette problématique se justifie d'autant plus que les études portant sur le contexte marocain sont rares (Abdelkhalek \& Solhi, 2009 et Mansouri \& Afroukh, 2009). En outre, elles ont considéré cinq banques sur la période 1993-2006, donc avant que les effets éventuels de la crise ne se fassent sentir sur les banques marocaines.

Dans ce sens, cette recherche se veut être un complément des résultats des études antérieures puisque la période d'analyse s’étend de 2004 à 2015 et le nombre de banques considéré est de huit au lieu de cinq. Par ailleurs, la focalisation sur les déterminants idiosyncratiques permet de tester d'autres variables non prises en compte par ces études telles que la structure de la propriété du capital (publique, privée ou filière d’une banque étrangère) ou sa cotation en bourse.

Pour ce faire, il serait opportun, dans un premier temps, de présenter les caractéristiques et les spécificités du système bancaire marocain, objet de l'étude. Cette présentation sera suivie, dans un deuxième temps, de la revue de la littérature afin de cerner le concept de performance bancaire et ses déterminants. Enfin, dans un dernier volet, et après spécification de la méthodologie de recherche, une analyse économétrique sur données de panel portera sur les huit banques universelles marocaines durant la période 20042015.

\section{Contexte de la recherche : caractéristiques et spécificités du système bancaire marocain}

L'analyse du système bancaire marocain et de son évolution permet de relever quatre traits saillants à savoir :

- $\quad$ c’est un système oligopolistique ;

- $\quad$ marqué par une forte concentration ;

- $\quad$ très régulé ;

- $\quad$ et résilient aux chocs. 


\section{Système oligopolistique}

L'analyse de l'évolution dans le temps du nombre d'établissements bancaires permet de déceler aisément le caractère oligopolistique du système bancaire marocain. En effet, et comme l'illustre le tableau n 1 , ce nombre est resté inchangé depuis 2009, se limitant à 19 banques. En outre, il faut signaler que parmi ces 19 banques figurent 11 banques régionales rattachées au groupe Crédit Populaire du Maroc (CPM). Ce qui limite le nombre de banques dites « universelles » à huit seulement.

Tableau $n^{\circ} 1$ : évolution du nombre d'établissements bancaires et autres organismes financiers

\begin{tabular}{|c|c|c|c|c|c|c|c|c|c|c|c|c|}
\hline & 2004 & 2005 & 2006 & 2007 & 2008 & 2009 & 2010 & 2011 & 2012 & 2013 & 2014 & 2015 \\
\hline Banques & 17 & 16 & 16 & 16 & 18 & 19 & 19 & 19 & 19 & 19 & 19 & 19 \\
\hline Banques à capital majoritairement étranger & 5 & 5 & 5 & 5 & 7 & 7 & 7 & 7 & 7 & 7 & 7 & 7 \\
\hline Banques à capital majoritairement public & 6 & 5 & 5 & 5 & 5 & 6 & 6 & 5 & 5 & 5 & 5 & 5 \\
\hline Banques à capital privé majoritairement marocain & 6 & 6 & 6 & 6 & 6 & 6 & 6 & 7 & 7 & 7 & 7 & 7 \\
\hline Sociétés de financement & 40 & 36 & 36 & 37 & 37 & 36 & 36 & 35 & 36 & 35 & 34 & 34 \\
\hline Sociétés de crédit à la consommation & 22 & 19 & 19 & 20 & 20 & 19 & 19 & 18 & 18 & 17 & 16 & 16 \\
\hline Sociétés de crédit-bail & 8 & 7 & 7 & 7 & 7 & 6 & 6 & 6 & 6 & 6 & 6 & 6 \\
\hline Sociétés de crédit immobilier & 2 & 2 & 2 & 2 & 2 & 2 & 2 & 2 & 2 & 2 & 2 & 2 \\
\hline Sociétés de cautionnement & 2 & 2 & 2 & 2 & 2 & 2 & 2 & 2 & 2 & 2 & 2 & 2 \\
\hline Sociétés d'affacturage & 2 & 2 & 2 & 2 & 2 & 2 & 2 & 2 & 2 & 2 & 2 & 2 \\
\hline Sociétés de gestion de moyens de paiement & 4 & 4 & 4 & 3 & 3 & 2 & 2 & 2 & 3 & 3 & 3 & 3 \\
\hline Autres sociétés & & & & 1 & 1 & 3 & 3 & 3 & 3 & 3 & 3 & 3 \\
\hline Nombre total d'établissements de crédit & 57 & 52 & 52 & 53 & 55 & 55 & 55 & 54 & 55 & 54 & 53 & 53 \\
\hline Banques offshore & 6 & 6 & 6 & 6 & 6 & 6 & 6 & 6 & 6 & 6 & 6 & 6 \\
\hline Associations de micro-crédit & 12 & 12 & 13 & 14 & 13 & 12 & 12 & 13 & 13 & 13 & 13 & 13 \\
\hline Sociétés de transfert de fonds & & & & & 7 & 9 & 8 & 10 & 10 & 9 & 9 & 9 \\
\hline Autres établissements & 3 & 3 & 3 & 3 & 3 & 2 & 2 & 2 & 2 & 2 & 2 & 2 \\
\hline Total & 78 & 73 & 74 & 76 & 84 & 84 & 83 & 85 & 86 & 84 & 83 & 83 \\
\hline
\end{tabular}

Source: établi à partir des rapports annuels sur la supervision bancaire publiés par Bank AlMaghrib.

Notons, toutefois, que cette situation tend à changer avec l'avènement des banques participatives suite à la promulgation de la loi bancaire de 2014 .

\section{Système marqué par une forte concentration}

La concentration du système bancaire marocain se reflète aussi bien au niveau géographique qu'au niveau des différents indicateurs d'activités. Ainsi, géographiquement, et sans surprise, l'axe Rabat-Casablanca se trouve en tête de liste en nombre de guichets, mais aussi en termes de dépôts et de crédits, comme l’illustre le graphique 1 ci-après : 
Graphique 1 : Part de chaque région dans le total réseau, dépôts et crédits (en \%), année 2015

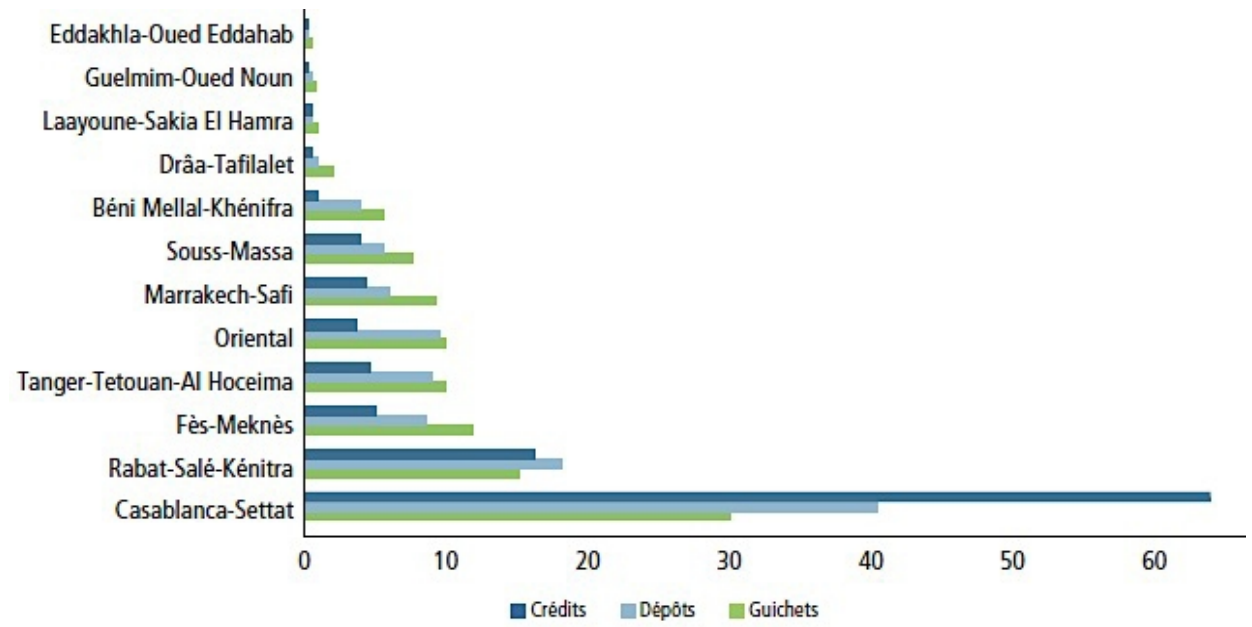

Source: BANK AL-MAGHRIB (2015). Rapport annuel sur la supervision bancaire, p. 14.

Par ailleurs, les trois premières banques marocaines, à savoir AttijariWafaBank (AWB), la Banque Marocaine du Commerce Extérieure (BMCE) et la Banque Centrale Populaire (BCP), concentrent à elles seules plus de $65 \%$ des dépôts, des crédits ainsi que du total actif, comme en témoignent les graphiques $2 \mathrm{a}, 2 \mathrm{~b}$ et $2 \mathrm{c}$ ci-dessous :

Graphique 2a

Concentration du total actif en \%

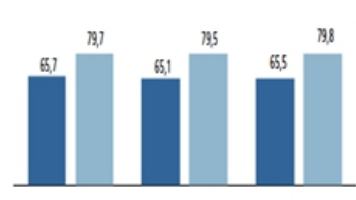

Graphique 2b

Concentration des crédits en \%

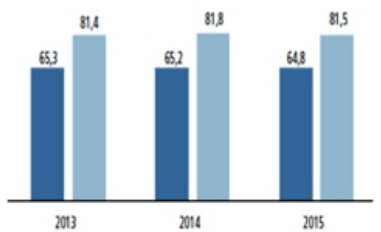

Graphique 2c

Concentration des dépôts en \%

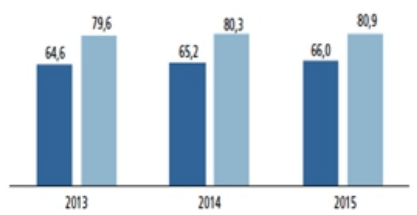

口 3 premiëres banques 5 premières banques

Source: BANK AL-MAGHRIB (2015). Rapport annuel sur la supervision bancaire, p.18.

Cette forte concentration s'explique d'une part, par la nature oligopolistique du système bancaire marocain comme signalé précédemment, et d'autre part, par la concentration géographique aussi bien de la population que de l'activité économique sur l'axe Rabat-Casablanca.

De même, le statut de l'actionnariat reste dominé par le capital privé marocain alors que les capitaux étrangers et publics demeurent minoritaires avec une légère tendance à la baisse pour les capitaux étrangers notamment en termes d'actif et de dépôts (voir graphique 3 ci-après). 
Graphique 3 : Concentration en fonction du statut de l'actionnariat (en \%)

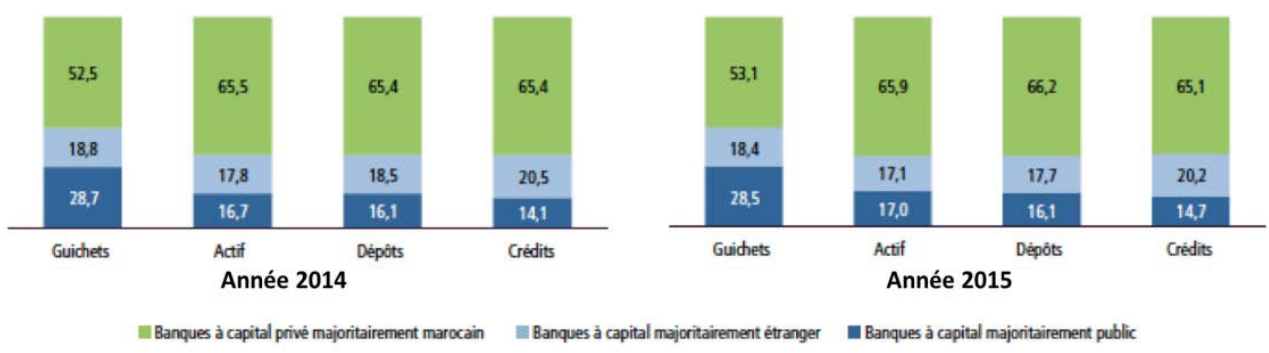

Source: BANK AL-MAGHRIB (2015). Rapport annuel sur la supervision bancaire, p.19.

\section{Système très régulé}

Les réformes radicales du système bancaire marocain ont débuté avec la loi bancaire de 1993 (loi n¹-93-147), qui avait alors constitué une rupture avec le système préexistant. Ainsi, « libéralisation » et " désencadrement » étaient les nouveaux mots d'ordre. En effet, la suppression des emplois obligatoires des banques, la levée de l'encadrement du crédit et la libéralisation des taux d'intérêt ont donné plus de marges de manœuvre aux banques, mais sans réduire pour autant la tutelle de Bank Al-Maghrib (BAM) et du ministère des finances.

Ainsi, si cette réforme s'est traduite par le décloisonnement du secteur en développant la "banque universelle », sa mise en place s'est accompagnée par le renforcement des règles prudentielles.

Ce processus s’est poursuivi avec la réforme de 2006 (loi n³4-03) qui a consacré l'autonomie de BAM et consolidé ses attributions (agréments, contrôle, sanctions). Cette réforme a également renforcé le dispositif réglementaire et prudentiel (Bâle II) ; et a poursuivi la refonte du cadre comptable des comptes consolidés, en conformité avec les normes IAS/IFRS. Cette mise à niveau avec les règles prudentielles internationales dénote du souci permanent des autorités de tutelle pour éviter tout dérèglement lié à la libéralisation et à l'ouverture du secteur.

L’année 2014 a également connu la promulgation de la dernière loi bancaire en date (loi $\mathrm{n}^{\circ} 103-12$ ) qui a introduit, après de longues tractations qui ont duré près de cinq ans, de nouveaux acteurs et services financiers à savoir les "banques participatives ». Ces dernières sont appelées à jouer un rôle important en offrant des services alternatifs au côté du système bancaire « classique ».

Cette loi a aussi défini le cadre légal pour l'exercice de la surveillance macro-prudentielle des risques systémiques. Ce qui va dans le sens du renforcement des règles prudentielles en vue de prévenir la survenue de crises pouvant affecter, non seulement le système bancaire, mais aussi l'économie dans son ensemble, comme ce fût le cas après la crise de 2008 pour la plupart des pays qui ont été fortement touchés. 
Dans le même ordre d'idées, la loi a prévu l'amélioration des mécanismes de résolution de crises et de traitement des difficultés bancaires ainsi que le renforcement de la protection de la clientèle, à travers la mise en place d'un fonds de garantie des dépôts.

\section{Système résilient}

Suite à la crise financière internationale de 2008, et en vue de préserver le système bancaire marocain et l'économie dans son ensemble contre les éventuels risques systémiques, BAM a assigné les banques dès le $1^{\mathrm{er}}$ juin $2010^{1}$ à se soumettre à des " stress tests ». Ces tests doivent couvrir l'ensemble des métiers de la banque et doivent être menés par chaque établissement bancaire à des intervalles réguliers (au moins une fois par an). En parallèle,

«Bank Al-Maghrib réalise régulièrement trois grandes catégories de stress tests afin d'évaluer la résilience du système bancaire face à des chocs extrêmes :

1 - Stress tests de sensibilité qui consistent à mesurer l'impact d'un choc de crédit, de liquidité, de marché, de taux d'intérêt ou de change, sur l'équilibre bilantiel et/ou la solvabilité des institutions financières.

2 - Stress tests de contagion interbancaire qui mesurent le risque de propagation de la défaillance d'une banque aux autres banques de la place à travers leurs engagements bilatéraux, sur les marchés interbancaires à blanc et collatéralisé.

3 - Stress tests macro qui évaluent la capacité du système bancaire à résorber des chocs émanant de l'environnement macroéconomique. » ${ }^{2}$

Par ailleurs, BAM a conduit deux exercices de simulation de crise ${ }^{3}$, le premier en 2009 et le deuxième en 2014, et ce avec l'appui de la Banque Mondiale et en collaboration avec le Ministère de l'Economie et des Finances et les autres autorités financières.

Les principales conclusions de ces stress tests sont plutôt rassurantes. En effet, face à des éventuels chocs macroéconomiques, le système bancaire marocain affiche une bonne résilience. De même, la vulnérabilité des banques face au risque de liquidité demeure très maîtrisable et leur exposition aux risques de contagion limitée. En revanche, les tests révèlent une forte exposition des banques au risque de concentration en cas de défaillance des plus grands débiteurs.

\footnotetext{
${ }^{1}$ Directive n²/G/2010 du 03 mai 2010.

${ }^{2}$ http://www.bkam.ma/Stabilite-financiere/Cadre-analytique/Stress-tests

${ }^{3}$ Tests du Financial Sector Assessment Program FSAP. Pour de plus amples détails, voir le Rapport sur la stabilité financière (2016) de BAM - ACAPS - AMMC.
} 


\section{Cadre théorique : mesures et déterminants de la performance bancaire Les mesures de la performance bancaire dans la littérature}

Dans la littérature, la performance bancaire demeure majoritairement appréhendée au travers d’indicateurs quantitatifs financiers (Yao, 2005 ; Heffernan \& Fu, 2010 ; Ongore \& Kusa, 2013). Les plus communément utilisés sont la marge nette d'intérêt (Net Interest Margin: NIM), la rentabilité des actifs (Return On Assets : ROA) et la rentabilité des capitaux propres (Return On Equity : ROE).

La NIM est généralement calculée en rapportant soit la marge d'intérêt ou le Produit Net bancaire (PNB) au Total Bilan ${ }^{4}$ (ou encore au Total Bilan moyen de deux années consécutives), soit :

$$
\mathrm{NIM}=\frac{\text { Marge d'intérêts ou PNB }}{\text { Total Bilan ou Total Bilan moyen }}
$$

Quant au ROA, il rapporte un indicateur de résultat (soit le résultat net de l'exercice RNE ou le Résultat avant impôts) au Total Bilan (ou encore au Total Bilan moyen de deux années consécutives, auquel cas l’appellation utilisée est ROAA : Return On Average Assets) :

$$
\text { ROA (ou ROAA) }=\frac{\text { RNE ou Résultat avant impôts }}{\text { Total Bilan ou Total Bilan moyen }}
$$

Enfin, le ROE qui exprime la rentabilité des capitaux propres (et sa variante ROAE : Return On Average Equity) est mesuré par le rapport entre le RNE et les Fonds Propres (FP) ou les FP moyens : $\mathrm{ROE}$ (ou ROAE) $=\frac{\mathrm{RNE}}{\text { FP ou FP moyens }}$

Demirgüç-Kunt et al. (1999), dans leur étude comparative portant sur 80 pays et couvrant la période 1988-1995, ont utilisé aussi bien la NIM que le ratio ROA (Return On Assets) pour appréhender la performance bancaire. Ce dernier ratio a été calculé en rapportant le Résultat avant impôts au Total Actif.

Pour leur part, Athanasoglou et al. (2008) ainsi que Dietricha \& Wanzenried (2009), ont eu recours aux ratios ROAA et ROAE comme approche de la performance dans leurs études portant respectivement sur les banques grecques et suisses.

Dans une étude plus élargie, Heffernan \& Fu (2010) ont comparé les régressions sur quatre variables dépendantes mesurant la performance à savoir la NIM, le ROAA, le ROAE et l'EVA ${ }^{5}$ (Economic Added Value). Sur la base des tests de signification des coefficients, les résultats suggèrent que

\footnotetext{
${ }^{4}$ Total Bilan et Total Actif sont équivalents

${ }^{5}$ Grosso modo, EVA = résultat économique - coût du capital investi (dettes et fonds propres). Sa mise en œuvre nécessite des retraitements en vue de passer du Résultat Comptable au Résultat Economique, ainsi qu'une évaluation du coût du capital.
} 
les meilleures variables dépendantes sont l'EVA et la NIM relativement aux ROAA et ROAE.

En revanche, l'EVA reste d'usage limité pour la mesure de la performance bancaire. En effet, étant initialement destinée à être utilisée par les entreprises industrielles et commerciales, son application aux banques nécessite beaucoup d'adaptations, hormis les retraitements nécessaires pour son opérationnalisation.

En conséquence, pour les besoins de notre étude, nous utiliserons la NIM comme variable dépendante.

\section{Les déterminants de la performance bancaire dans la littérature}

L'analyse des déterminants de la performance bancaire a fait l'objet de plusieurs études, menées aussi bien au niveau d'un pays déterminé (Athanasoglou et al., 2008 ; Dietricha \& Wanzenried, 2009 ; Heffernan \& Fu, 2010 ; Ongore \& Kusa, 2013) qu'au niveau international (Bourke, 1989; Molyneux \& Thornton, 1992 ; Demirgüç-Kunt et al., 1999 ; Yao, 2009 ; Bordeleau \& Graham, 2010 ; Nguyen, 2014).

Ces déterminants sont globalement classés en facteurs idiosyncratiques et en facteurs externes. Les premiers désignent les variables spécifiques internes qui influencent la performance des banques et sont majoritairement inspirés du cadre CAMEL ${ }^{6}$. Il s'agit notamment du capital, de la qualité de l'actif, du système de management de la banque, des résultats et de la liquidité. D'autres facteurs comme la taille et le contrôle sont aussi largement discutés dans la littérature. Le tableau $n^{\circ} 2$ qui suit reprend les principaux déterminants internes et les mesures qui y sont fréquemment associées :

\footnotetext{
${ }^{6}$ Acronyme de « Capital adequacy, Asset quality, Management, Earnings and Liquidity », CAMEL est à la base un système d'estimation et de prévision du risque de défaut bancaire élaboré aux Etats-Unis au début des années 1980. Son utilisation a ensuite été largement étendue à la problématique d'évaluation et d'identification des déterminants de la performance bancaire. Notons que dans le cadre d'une analyse des déterminants, les résultats sont considérés comme variable dépendante.
} 
Tableau $\mathrm{n}^{\circ} 2$ : Principaux déterminants internes et mesures y associées

\begin{tabular}{|c|c|}
\hline $\begin{array}{l}\text { Principaux } \\
\text { déterminants } \\
\text { internes }\end{array}$ & Mesures fréquemment utilisées \\
\hline La taille & $\begin{array}{l}\text { Total Bilan ou son logarithme } \\
\text { Part de marché }=\frac{\mathrm{PEB}_{\mathrm{i}}}{\sum_{\mathrm{i}=1}^{\mathrm{n}} \mathrm{PEB}} \\
\text { Avec }: \mathrm{PEB}_{\mathrm{i}} \text { est le produit d'exploitation bancaire de la banque i. Il est } \\
\text { l'équivalent du chiffre d'affaires pour les entreprises non financières. Il } \\
\text { est rapporté à la somme des PEB des banques de l'échantillon. } \\
\text { Une autre variante de la part de marché consiste à rapporter le total bilan } \\
\text { de la banque } \mathrm{i}\left(\mathrm{TB}_{\mathrm{i}}\right) \text { à la somme des TB des banques de l'échantillon. }\end{array}$ \\
\hline $\begin{array}{c}\text { La } \\
\text { capitalisation } \\
\end{array}$ & Ratio de capitalisation $=\frac{\text { Fonds Propres }}{\text { Total Bilan }}$ \\
\hline La liquidité & $\frac{\text { Créances totales }}{\text { Total Bilan }}$ ou $\frac{\text { Créances totales }}{\text { Dépôts clients }}$ ou $\frac{\text { Créances à court terme }}{\text { Passifs à court terme }}$ \\
\hline $\begin{array}{l}\text { La qualité du } \\
\text { crédit }\end{array}$ & $\frac{\text { Créances En Souffrances (CES) }}{\text { Créances totales }}$ ou $\frac{\text { CES }}{\text { Créances clients }}$ \\
\hline $\begin{array}{c}\text { L’efficacité } \\
\text { opérationnelle }\end{array}$ & $\frac{\text { Coûts opérationnels }}{\text { Résultat net ou opérationnel }}$ ou $\frac{\text { Coûts opérationnels }}{\text { Total Bilan }}$ \\
\hline Le contrôle & $\begin{array}{l}\text { Pourcentage majoritaire dans le capital social : } \\
\text { privé vs public ou national vs étranger }\end{array}$ \\
\hline
\end{tabular}

Quant aux facteurs externes, ils font référence aux variables macroéconomiques susceptibles d'impacter la stabilité et la résilience du système bancaire dans son ensemble, mais aussi la performance des banques. La croissance du PIB, les taux d'imposition, la structure/concentration du marché bancaire, le taux d'inflation et les taux d'intérêt sont abondamment relayés dans la littérature. Notons que ces variables sont généralement plus pertinentes dans le cadre de comparaisons internationales. Leur impact serait ressenti sur le système bancaire dans son ensemble, et conséquemment, il serait difficile d'expliquer les différences de performances entre les banques (prises individuellement) inhérentes à chaque variable externe. C'est la raison pour laquelle nous les avons écarté dans cette étude, d'autant plus qu'au Maroc, des facteurs comme les taux d'intérêt et d'imposition sont encore fixés par l'Etat. C'est ce qui explique leur stabilité, voire constance, sur la période d'analyse choisie de 2004 à 2015.

Or, si la majorité de ces études s'accordent sur la classification des déterminants de la performance, les résultats obtenus sont souvent différents voire contradictoires. Ces divergences sont accentuées par le fait que les études en question sont menées dans des pays, des environnements et des périodes de temps qui diffèrent (Athanasoglou et al., 2008).

Ainsi, s'agissant de l'impact de la taille sur la performance des banques, un consensus est loin d'être atteint. Certaines études concluent à un 
effet significativement positif de la taille, en ce sens que les banques de taille moyenne à grande seraient plus performantes (Short, 1979 ; Bourke,1989 ; Molyneux \& Thornton,1992). D’autres recherches arguent que plus la taille de la banque augmente, moins elle est efficiente en raison des coûts de gestion qui deviennent plus élevés (Berger et al.,1987 ; Kasman et al., 2010). Pour d'autres auteurs en revanche, le lien entre la taille de la banque et sa performance ne semble pas très significatif (Goddard et al, 2004 et Athanasoglou et al., 2008).

Concernant la capitalisation, les résultats de la majorité des études empiriques concordent quant à l'existence d'un lien positif probant entre celle-ci et la performance d'une banque. En effet, les banques avec des ratios de fonds propres plus élevés ont tendance à bénéficier d'une baisse du coût du financement en raison des risques de faillite potentiels faibles.

Le même consensus parmi les études susmentionnées est constaté lors de l'analyse de l'impact de la qualité du crédit et de l'efficacité opérationnelle sur la performance bancaire. Ce sont également deux indicateurs étroitement liés au risque de défaillance, et par conséquent, leur amélioration $^{7}$ affecte positivement la performance. A noter, dans ce sens, que la variable "qualité du crédit » est généralement considérée comme une mesure directe du risque bancaire.

Néanmoins, la relation entre la liquidité et la rentabilité bancaire divise les auteurs. Si certaines études aboutissent à une relation positive entre les liquidités et la rentabilité bancaire (Bourke, 1989), d'autres trouvent une relation négative mitigée entre les deux variables (Molyneux \& Thornton, 1992 ; Goddard et al. 2004). Si pour le premier groupe la détention de liquidités suffisantes constitue un matelas de sécurité contre les risques éventuels, les détracteurs de cet argument avancent que trop de liquidités représente un manque à gagner pour la banque et, par voie de conséquence, impacterait négativement sa performance.

En ce qui concerne le contrôle, la plupart des études ayant considéré cette variable ont opposé les banques privées aux banques publiques/nationalisées. Si globalement, les banques privées affichent des performances meilleures que celles publiques, les conclusions diffèrent selon le contexte étudié et la période d'analyse. En effet, cette différence de performance s'accentue dans les pays en développement et particulièrement ceux marqués par un contexte politique spécifique comme la corruption ou pendant les périodes de crises (Micco et al., 2007 ; Million Cornett et al. 2010). De leur côté, Dietricha \& Wanzenried (2009), tout en affirmant que

\footnotetext{
${ }^{7}$ L'amélioration dans le cas de ces deux ratios doit être entendue comme leur baisse. En effet, la qualité du crédit s’améliore si la part des créances en souffrance baisse. De même, l'efficacité opérationnelle est meilleure quand la part des charges diminue. Donc, il faut être vigilant lors de leur interprétation.
} 
les banques privées sont légèrement plus rentables que celles détenues par l'Etat, trouvent que les différences de performance sont plus marquées entre les banques étrangères (nettement moins rentables) et les banques suisses.

\section{Approche méthodologique : analyse sur données de panel Spécification du modèle}

A l'instar de la plupart des études empiriques relatives à la performance bancaire et réalisées sur données de panel, nous adoptons l'approche méthodologique de Short (1979) reprise par Bourke (1989), Molyneux \& Thornton (1992), Demirgüç-Kunt \& Huizinga (1999) et Goddard et al. (2004).

Il s’agit d'une régression linéaire sur données de panel qui met en relation une variable dépendante (dans notre cas la performance de la banque) avec un ensemble de variables explicatives (déterminants).

$$
\operatorname{PERF}_{\mathrm{i}, \mathrm{t}}=c+\sum_{j=1}^{n} \alpha_{j} X_{i, t}^{j}+\varepsilon_{i, t}
$$

$\mathrm{PERF}_{\mathrm{i}, \mathrm{t}}$ : la performance de la banque i pour l'année $\mathrm{t}$

$c$ : la constante,

$X_{i, t}^{j}$ : les variables indépendantes

et $\boldsymbol{\varepsilon}_{\boldsymbol{i}, \mathrm{t}}$ représente l'erreur

Les données de panel présentent l'avantage de croiser les séries chronologiques aux données en coupe instantanée pour les entités observées, ce qui permet de disposer d'un nombre plus important d'observations.

Toutefois, l'utilisation des données de panel soulève toujours la question du choix de la spécification du modèle en optant pour un modèle à effets fixes ou à effets aléatoires ${ }^{8}$. Givord \& Guillerm (2016) arguent que :

"Les micro-économètres tentent en général de mettre en évidence l'effet causal d'une variable explicative, et les effets de contexte ne sont considérés que comme des "nuisances"...dont il faut se débarrasser. Les modèles à effets fixes sont plus adaptés à cet objectif que les modèles à effets aléatoires. Les modèles à effets fixes en particulier ne reposent pas sur l'indépendance entre ces effets non observés...et des variables explicatives, contrairement aux modèles à effets aléatoires. » p.15.

Ainsi, le modèle à effets fixes semble plus adapté à notre problématique. Le traitement des données a été réalisé avec «IBM SPSS Statistics 20 » en utilisant la procédure des Modèles Mixtes Linéaires (MML) avec effets fixes et en utilisant l'estimation par maximum de vraisemblance

${ }^{8}$ Pour une explication détaillée des hypothèses sous-jacentes à chacun des deux modèles ainsi que des critères de choix, voir Givord \& Guillerm (2016). 
restreint (restricted estimator maximum likelihood, REML). Cette dernière permet de dépasser la contrainte d’homoscédasticité nécessaire pour une régression avec les Moindres Carrés Ordinaires (MCO). De ce fait, « elle est jugée préférable, car elle produit des estimateurs plus fiables lorsque les effectifs par groupe sont faibles » (Givord \& Guillerm, 2016, p. 28).

\section{Choix des variables et hypothèses sous-jacentes}

Comme signalé précédemment (voir 2.1.), la NIM constitue la meilleure approximation de la performance bancaire relativement au ROAA et au ROAE. Nous avons privilégié un ratio qui lui est très similaire adopté par BAM, celui de la Marge Bancaire Globale (MBG) calculée ainsi :

$$
\mathrm{MBG}=\frac{\text { Produit Net Bancaire (PNB) }}{\text { Total Bilan moyen }}
$$

En effet, le PNB est composé majoritairement de la marge d'intérêt stricto sensu ${ }^{9}$ à laquelle se rajoutent les marges sur commissions, les résultats sur opérations de crédit-bail et de location et les résultats des opérations de marché.

Ainsi, le PNB est une notion moins restrictive que la marge d'intérêt et donc plus représentative de l'activité principale de la banque. En outre, le choix de la MBG comme variable dépendante permet de s'aligner sur les calculs de BAM pour des éventuels rapprochements.

Par ailleurs, les tests statistiques de normalité permettent d'appuyer ce choix comme l'illustre le tableau $n^{\circ} 3$ suivant :

Tableau n³ : Tests de normalité

\begin{tabular}{|c|c|c|c|c|c|c|}
\hline & \multicolumn{3}{|c|}{ Kolmogorov-Smirnov $^{\text {a }}$} & \multicolumn{3}{c|}{ Shapiro-Wilk } \\
\cline { 2 - 7 } & Statistique & ddl & Signification & Statistique & ddl & Signification \\
\hline MBG &, 055 & 96 &, $200^{*}$ &, 983 & 96 &, 253 \\
ROAA &, 281 & 96 &, 000 &, 440 & 96 &, 000 \\
ROAE &, 370 & 96 &, 000 &, 419 & 96 &, 000 \\
\hline
\end{tabular}

*. Il s'agit d'une borne inférieure de la signification réelle

a. Correction de signification de Lilliefors

Ainsi, les p-valeurs $>0,05$ pour la MBG des tests de K-S et de S-W permettent de retenir l'hypothèse de normalité pour la MBG et son rejet aussi bien pour le ROAA que le ROAE dont les p-valeurs<0,05. Donc, la MBG remplit la condition nécessaire de l'utilisation d'une régression linéaire.

Quant aux variables explicatives qui seront intégrées dans le modèle, rappelons que l'analyse a été délibérément limitée aux déterminants

\footnotetext{
${ }^{9}$ Dans l'état des soldes de gestion bancaire marocain, la marge d'intérêt = intérêts perçus et produits assimilés-charges d'intérêts et assimilées. Elle représente près de 2/3 du PNB.
} 
idiosyncratiques dans le but d'isoler l'impact de ces facteurs sur la performance bancaire.

Ces variables ont été sélectionnées à partir de la revue de la littérature, mais aussi après un test de corrélation avec la variable dépendante. Il s’agit de la taille, de la capitalisation, de l'efficacité opérationnelle, de la liquidité, du coût de financement, de la qualité du crédit, du contrôle et de la cotation. Notons que cette dernière variable n'est pas très documentée dans les études passées en revue, mais nous avons choisie de l'intégrer puisque la population étudiée est composée aussi bien de banques cotées que celles non cotées.

Signalons, par ailleurs, que l’indicateur privilégié pour appréhender la taille est la part de marché et non le total bilan, et ce pour deux raisons : premièrement, la part de marché est un ratio et donc exprime mieux les différences relatives; deuxièmement, vue la concentration du système bancaire et comme le TB est une grandeur cumulative, les mêmes banques se retrouveront au même rang chaque année, ce qui va occulter les différences de performances annuelles.

Le tableau $n^{\circ} 4$ qui suit reprend l'ensemble des variables explicatives avec leur mode de calcul et les hypothèses associées :

Tableau $\mathrm{n}^{\circ} 4$ : variables explicatives et hypothèses sous-jacentes

\begin{tabular}{|c|c|c|}
\hline $\begin{array}{c}\text { Variables } \\
\text { explicatives }\end{array}$ & Mesure adoptée & $\begin{array}{c}\text { Hypothèses } \\
\text { (Impact attendu sur la performance) }\end{array}$ \\
\hline $\begin{array}{l}\text { La taille : Part de } \\
\text { marché }\end{array}$ & $\mathrm{PDM}=\frac{\mathrm{PEB}_{\mathrm{i}}}{\sum_{\mathrm{i}=1}^{\mathrm{n}} \mathrm{PEB}}$ & La performance augmente avec la taille \\
\hline Capitalisation & CAP_RA $=\frac{\text { Fonds Propres }}{\text { Total Bilan }}$ & $\begin{array}{l}\text { La performance augmente avec le } \\
\text { renforcement de la capitalisation }\end{array}$ \\
\hline $\begin{array}{c}\text { Efficacité } \\
\text { opérationnelle }\end{array}$ & $\begin{array}{l}\text { EFFIC } \\
=\frac{\text { Charges Générales d'Exploitation }}{\text { PNB }}\end{array}$ & $\begin{array}{c}\text { Plus le ratio augmente (inefficacité) et } \\
\text { plus la performance baisse }\end{array}$ \\
\hline $\begin{array}{l}\text { Liquidité mesurée } \\
\text { par deux ratios }\end{array}$ & $\begin{array}{l}\text { LIQ_GL }=\frac{\text { Créances totales }}{\text { Dépôts clients }} \\
\text { LIQ_RDT }=\frac{\text { Créances à court terme }}{\text { Passifs à court terme }}\end{array}$ & $\begin{array}{l}\text { Lien positif entre les ratios de liquidité } \\
\text { et la performance }\end{array}$ \\
\hline $\begin{array}{c}\text { Coût de } \\
\text { financement }\end{array}$ & COU_FIN $=\frac{\text { Charges d’intérêt }}{\text { Dépôts clients }}$ & $\begin{array}{c}\text { L'augmentation des coûts implique une } \\
\text { augmentation de la MBG (répercussion } \\
\text { du coût de financement sur les intérêts } \\
\text { perçus) }\end{array}$ \\
\hline Qualité du crédit & QUAL $=\frac{\text { CES }}{\text { Créances clients }}$ & $\begin{array}{c}\text { Plus le ratio augmente (risque élevé) et } \\
\text { plus la performance baisse }\end{array}$ \\
\hline Contrôle & $\begin{array}{l}\text { Catégorielle : } \\
\text { Public : } 0 \\
\text { Privé : } 1 \\
\text { Etranger : } 2\end{array}$ & $\begin{array}{l}\text { Les banques étrangères seraient plus } \\
\text { performantes que les banques } \\
\text { marocaines privées. Ces dernières } \\
\text { seraient plus performantes que les } \\
\text { banques publiques }\end{array}$ \\
\hline Cotation & $\begin{array}{l}\text { Dichotomique : } \\
\text { Non cotée : } 0 \\
\text { Cotée }: 1\end{array}$ & $\begin{array}{l}\text { Les banques cotées seraient plus } \\
\text { performantes }\end{array}$ \\
\hline
\end{tabular}




\section{Les données}

Les données utilisées dans cette recherche sont relatives à huit banques universelles marocaines pour la période 2004-2015, soit au total 96 observations pour chacune des variables retenues dans l'analyse. Les banques en question sont: AttijariWafaBank (AWB), la Banque Centrale Populaire (BCP), la Banque Marocaine du Commerce Extérieur (BMCE Bank), la Banque Marocaine du Commerce et de l'Industrie (BMCI), le Crédit Du Maroc (CDM), le Crédit Immobilier et Hôtelier (CIH), le Crédit Agricole du Maroc (CAM) et la Société Générale Marocaine des Banques (SGMB).

Les grandeurs brutes ont été extraites des états de synthèse annuels de toutes les banques, puis les ratios choisis comme variables explicatives ont été calculés. Les données étant relevées pour la même période sans observations manquantes, l'échantillon est donc équilibré (balanced). Les caractéristiques des variables de l'échantillon sont reprises dans les tableaux $n^{\circ} 5 a$ et $5 b$ suivants :

Tableau $\mathrm{n}^{\circ} 5 \mathrm{a}$ : Informations sur les variables catégorielles

\begin{tabular}{|l|c|c|c|c|}
\hline \multicolumn{2}{|c|}{} & $\mathrm{N}$ & Pourcentage \\
\hline \multirow{3}{*}{ Facteur } & \multirow{3}{*}{ Cotation } & NON & 24 & $25,0 \%$ \\
\cline { 3 - 4 } & & OUI & 72 & $75,0 \%$ \\
\cline { 3 - 4 } & \multirow{3}{*}{ Contrôle } & Total & 96 & $100,0 \%$ \\
\cline { 3 - 4 } & & Public & 34 & $35,4 \%$ \\
\cline { 3 - 4 } & & Privé & 26 & $27,1 \%$ \\
\cline { 3 - 5 } & Etranger & 36 & $37,5 \%$ \\
\cline { 3 - 5 } & & Total & 96 & $100,0 \%$ \\
\hline
\end{tabular}

Tableau n5b : Informations sur les variables continues

\begin{tabular}{|c|c|c|c|c|c|c|}
\hline \multicolumn{2}{|c|}{} & $\mathrm{N}$ & Minimum & Maximum & Moyenne & Ecart-type \\
\hline Variable dépendante & MBG & 96 & 1,18 & 6,26 & 3,9443 & 1,04342 \\
\hline \multirow{4}{*}{$\begin{array}{c}\text { Variables } \\
\text { indépendantes }\end{array}$} & PDM_CA & 96 & 3,83 & 31,86 & 12,4954 & 7,50911 \\
\cline { 2 - 7 } & EFFIC & 96 & 34,30 & 114,15 & 53,9670 & 13,73231 \\
\cline { 2 - 7 } & CAP_RA & 96 & $-6,04$ & 14,29 & 8,0264 & 3,38598 \\
\cline { 2 - 7 } & LIQ_GL & 96 & 88,34 & 1688,28 & 174,4888 & 229,57379 \\
\cline { 2 - 7 } & LIQ_RDT & 96 & 33,54 & 134,56 & 86,0283 & 23,86329 \\
\cline { 2 - 7 } & COU_FIN & 96 &, 84 & 3,22 & 1,5689 &, 41832 \\
\cline { 2 - 7 } & QUAL & 96 &, 63 & 59,51 & 10,7755 & 10,11764 \\
\hline
\end{tabular}

\section{Résultats et discussions}

Les principaux résultats de la régression sur données de panel avec le modèle à effets fixes sont repris dans le tableau $n^{\circ} 6$ ci-après : 
Tableau $^{\circ} 6$ : Estimations des effets fixes ${ }^{\mathrm{a}}$

\begin{tabular}{|c|c|c|c|c|c|c|c|}
\hline & & & & & & \multicolumn{2}{|c|}{$\begin{array}{c}\text { Intervalle de } \\
\text { confiance 95\% }\end{array}$} \\
\cline { 5 - 8 } Paramètres & Estimation & $\begin{array}{c}\text { Erreur } \\
\text { standard }\end{array}$ & $\mathrm{ddl}$ & $\mathrm{t}$ & Sig. & $\begin{array}{c}\text { Borne } \\
\text { inférieure }\end{array}$ & $\begin{array}{c}\text { Borne } \\
\text { supérieure }\end{array}$ \\
\hline Constante & 5,210928 &, 443175 & 21,560 & 11,758 &, 000 & 4,290751 & 6,131105 \\
[COTE=0] &, 532284 &, 094886 & 10,938 & 5,610 &, 000 &, 323297 &, 741270 \\
[COTE=1] & $0^{\mathrm{b}}$ & 0 & & & & & \\
[CONTRL=0] & $-1,002525$ &, 099737 & 8,176 & $-10,052$ &, 000 & $-1,231659$ &,- 773391 \\
[CONTRL=1] &,- 072321 &, 174359 & 26,535 &,- 415 &, 682 &,- 430370 &, 285728 \\
[CONTRL=2] & $0^{\mathrm{b}}$ & 0 & & & & & \\
PDM_CA &,- 033755 &, 010174 & 35,304 & $-3,318$ &, 002 &,- 054402 &,- 013108 \\
EFFIC &,- 021175 &, 006018 & 53,397 & $-3,518$ &, 001 &,- 033244 &,- 009106 \\
CAP_RA &, 059798 &, 015123 & 12,733 & 3,954 &, 002 &, 027057 &, 092538 \\
LIQ_GL &,- 000614 &, 000494 & 14,751 & $-1,243$ &, 233 &,- 001669 &, 000441 \\
LIQ_RDT &,- 014547 &, 002191 & 9,561 & $-6,638$ &, 000 &,- 019460 &,- 009633 \\
COU_FIN &, 451507 &, 132565 & 14,641 & 3,406 &, 004 &, 168347 &, 734666 \\
QUAL &, 055521 &, 007221 & 40,511 & 7,689 &, 000 &, 040932 &, 070109 \\
\hline
\end{tabular}

a. Variable dépendante : Marge Bancaire Globale=PNB/TBmoyen .

b. Ce paramètre est réglé sur zéro car il est redondant.

Ainsi, et comme en attestent les p-valeurs, tous les déterminants sélectionnés ont un impact significatif sur la performance des banques marocaines étudiées à l'exception du ratio de liquidité générale.

Concernant la cotation, et contrairement à l'hypothèse formulée, ce sont les banques non cotées qui affichent les meilleures performances. Ce résultat, surprenant au premier abord, reflète la spécificité du contexte marocain qui est caractérisé non seulement par un système bancaire oligopolistique et concentré, mais aussi par un marché boursier peu développé. Ce résultat est également à nuancer par le fait que parmi les huit banques étudiées, seules deux ne sont pas cotées (le CAM et la SGMB).

S'agissant de l'influence de la propriété du capital sur la performance bancaire, les résultats montrent que les banques à capital majoritairement privé (qu'il soit marocain [1]) ou étranger [2]) sont plus performantes que celles dont le capital est détenu majoritairement par l'Etat [0]. Ceci peut s'expliquer doublement : d'une part, les banques privées ont des méthodes de gestion plus efficientes notamment sur le plan de la maitrise des coûts ; et d'autre part, les banques publiques (notamment le CAM) se positionnent sur des créneaux où leur objectif premier est de proposer des financements avantageux pour certains secteurs d'activité. Ainsi, la combinaison de marges réduites à des coûts de gestion plus élevés permet d'élucider leur faible performance par rapport aux banques privées. En revanche, les résultats ne montrent pas de différences notoires entre les banques privées marocaines et étrangères.

Par ailleurs, dans le contexte marocain, la performance est inversement reliée à la taille de la banque, qui rappelons-le est mesurée par la 
part de marché. Ceci indique que les banques ayant les parts de marchés les plus importantes affichent une performance inférieure à celles disposant de parts de marché plus faibles. Deux explications peuvent être avancées :

Primo, l'accroissement de la part de marché peut être la résultante d'une stratégie de croissance agressive qui se fait au détriment des marges. Autrement dit, les banques acceptent des marges faibles pour accroitre leurs parts de marché ;

- $\quad$ Secundo, les grandes structures génèrent des frais de gestion plus élevés qui se répercutent négativement sur leurs marges.

En outre, l'efficacité opérationnelle s’est révélée un facteur important de la performance bancaire. La relation inverse du ratio choisi avec la MBG indique que les banques qui maitrisent leurs charges affichent des performances meilleures. Ce qui corrobore les résultats de la majorité des études empiriques passées en revue.

L'effet de la capitalisation sur la performance est positif, en ce sens que les banques ayant les ratios de capitalisation les plus élevées sont aussi les plus performantes. Ainsi, comme le suggère la littérature, le renforcement des fonds propres facilite le financement à moindre coût, et par conséquent une amélioration des marges, car il est perçu comme un signe d'une bonne santé financière de l’institution.

Quant à la liquidité, et contrairement aux résultats attendus, elle semble affecter négativement la performance des banques marocaines, notamment la détention de créances à court terme. En effet, le ratio de liquidité réduite impacte de manière plus significative la MBG comparé au ratio de liquidité générale. Ce qui va dans le sens des études qui soutiennent qu'une surliquidité représente un manque à gagner pour la banque (Molyneux \& Thornton, 1992 ; Goddard et al. 2004). C'est aussi la conclusion de Mansouri \& Afroukh (2009) concernant les banques marocaines : "la surcapitalisation des banques marocaines a entraîné une situation de surliquidité qui n’a profité de ce fait ni à la rentabilité globale ni aux marges d'intérêt bancaires. », p.16.

En revanche, la relation positive entre le coût de financement et la MBG s'est confirmée. Ainsi, toute augmentation du coût de financement serait systématiquement répercutée sur les clients.

Même constat concernant la relation entre la MBG et le ratio de la qualité du crédit. En effet, l'hypothèse d'une relation inverse ne s'est pas vérifiée, ce qui implique qu'une augmentation de la part des créances en souffrance (CES) s'accompagne d'une croissance de la MBG. Ceci laisse présager que face à l'accroissement des CES, les banques augmentent leurs marges pour compenser l'éventuel risque de défaut. Cette solution est d'autant plus envisageable que le système bancaire marocain tel que décrit précédemment reste très oligopolistique et fortement concentré. 


\section{Conclusion}

L'objectif de cette étude était d'analyser les déterminants idiosyncratiques de la performance des banques marocaines. Ce choix était motivé d'un côté par l'insuffisance des recherches dans ce domaine et d'un autre côté par l'intérêt d'étudier les facteurs internes spécifiques indépendamment des variables macroéconomiques, non que ces dernières ne soient pas signifiantes, mais elles ont plus d'intérêt dans le cadre de comparaisons internationales.

L’étude a été menée sur les données de huit banques marocaines de 2004 à 2015, ce qui justifie le recours à l'analyse en données de panel à l'instar de la majorité des études empiriques réalisées dans ce sens. Les variables dépendante et indépendantes ont également été sélectionnées à la lumière de la revue de la littérature.

Les résultats obtenus sont conformes aux hypothèses émises ainsi qu’à la littérature en ce qui concerne l'effet de la capitalisation, du coût de financement, de l'efficacité opérationnelle et de la nature du contrôle sur la performance bancaire.

En revanche, les effets de la taille, de la qualité du crédit, de la cotation et du ratio de liquidité réduite sont spécifiques aux banques marocaines. En effet, les tests statistiques révèlent un lien probant entre ces variables et la MBG mais qui va à l'opposé des hypothèses retenues initialement.

Enfin, l’impact du ratio de liquidité générale sur la performance bancaire s’est révélé non significatif.

Ces conclusions peuvent s’avérer utiles pour le choix des variables d'action susceptibles d'améliorer l'indicateur de performance bancaire étudié à savoir la MBG.

Ceci étant, et à l'instar de toute étude empirique, cette recherche ne peut prétendre à l'exhaustivité. Certains points non explorés peuvent constituer des pistes de recherches intéressantes notamment :

- La prise en compte du phénomène de la persistance de la performance en intégrant une variable retardée dans le modèle spécifié ;

- La prise en compte de variables liées à la qualité du management qui pourrait éclairer davantage la compréhension de la performance bancaire dans le contexte marocain ;

- La comparaison avec d’autres pays sera également plus instructive sur les spécificités du contexte marocain.

\section{References:}

1. Abdelkhalek, T. \& Solhi, S. (2009). Efficience et Productivité des Banques Commerciales Marocaines : Approche non Paramétrique. Economic Research Forum ERF, Working Paper n466. 
2. Aglietta, M. et Brand, T. (2015). La stagnation séculaire dans les cycles financiers de longue période. In Bensidoun, I. et CouppeySoubeyran, J. (Dir.) «L'économie mondiale 2016 » Éditions La Découverte, collection Repères, Paris, pp. 24-39.

3. Athanasoglou, P. P., Brissimis, S. N., \& Delis, M. D. (2008). Bankspecific, industry specific and macroeconomic determinants of bank profitability. Journal of international financial Markets, Institutions and Money, 18(2), 121-136.

4. Berger, A., Hanweck, G., Humphrey, D.B., (1987). Competitive viability in banking: Scale, scope and product mix economies. Journal of Monetary Economics 20(3), 501-520.

5. Bordeleau, E. \& Graham, C., 2010. The Impact of Liquidity on Bank Profitability. Bank of Canada Working Paper 2010-38, ISSN 17019397.

6. Bourke, P. (1989). Concentration and other determinants of bank profitability in Europe, North America and Australia. Journal of Banking \& Finance, 13(1), 65-79.

7. Deisting, F., Makhlouf, F. et Naamane, A. (2012). Développement financier, flux financiers et croissance économique. Centre d'Analyse Théorique et de Traitement des données économiques, Working Paper series.

8. Demirgüç-Kunt, A., \& Huizinga, H. (1999). Determinants of commercial bank interest margins and profitability : Some international evidence. The World Bank Economic Review, 13(2), 379-408.

9. Dietricha, A. \& Wanzenried, G. (2009). What Determines the Profitability of Commercial Banks? New Evidence from Switzerland. $12^{\text {th }}$ Conference of the Swiss Society for Financial Market Research, Genève, Suisse.

10. Easterly, W. (1993). How Much Do Distortions Affect Growth? Journal of Monetary Economics, 32(4), 187-212.

11. Givord, P. \& Guillerm, M. (2016). Les modèles multiniveaux. Série des documents de travail " Méthodologie Statistique » de l'INSEE, Document de travail M 2016/05.

12. Goddard, J., Molyneux, P. \& Wilson, J. O. S. (2004). The profitability of european banks: a cross-sectional and dynamic panel analysis. The Manchester School, 72(3), 363-381.

13. Heffernan, S. \& Fu, X. (2010). Determinants of financial performance in Chinese banking. Applied Financial Economics, 20(20), 15851600 .

14. Kasman, A., Tunc, G., Vardar, G. \& Okan, B. (2010). Consolidation and Commercial bank net interest margins: evidence from the old and 
new European union members and candidate countries. Economic Modeling, 27(3), 648-655.

15. King, R. G. \& R. Levine (1993). Finance and Growth: Schumpeter Might Be Right. Quarterly Journal of Economics, 108, 717-737.

16. Levine, R. (1997). Financial Development and Economic Growth: Views and Agenda. Journal of Economic Literature, 35, 688-726.

17. Levine, R. (2004). Finance and Growth: Theory and Evidence. NBER Working Paper Series $n^{\circ} 10766$, Cambridge, MA.

18. Lordon, F. (2009). Après la crise financière : « réguler » ou refondre ? Revue de la régulation [En ligne], 5 | 1er semestre / Spring 2009. URL : http://regulation.revues.org/7461.

19. Mansouri, B. \& Afroukh, S. (2009). La Rentabilité des Banques et ses Déterminants : Cas du Maroc. Economic Research Forum ERF, Working Paper $n^{\circ} 462$.

20. Micco, A., Panizza, U., Yanez, M. (2007). Bank ownership and performance. Does politics matter? Journal of Banking and Finance, 31(1), 219-241.

21. Millon Cornett, M., McNutt, J.J., Tehranian, H. (2010). The financial crisis, internal corporate governance, and the performance of publicly-traded U.S. bank holding companies. SSRN, Working Paper Series.

22. Molyneux, P. \& Thornton, J. (1992). Determinants of European bank profitability: A note. Journal of banking and finance, 16, 1173-1178.

23. Nguyen, T-D-L., 2014. Liquidité, Risque et Profit des banques: Application aux systèmes bancaires de pays de la zone euro. CREM, Université de Rennes 1, France.

24. OCDE (2009). Réponse stratégique de l'OCDE à la crise financière et économique : Contributions à l'effort mondial.

25. Reinhart C. \& Rogoff K. (2009). The aftermath of financial crises. NBER Working Paper, $n^{\circ} 14656$, Cambridge, MA.

26. Short, B., (1979). The relation between commercial bank profit rates and banking concentration in Canada, Western Europe and Japan. Journal of Banking and Finance, 3(3), 209-219.

27. Stiglitz, J. E. (2010a). Le triomphe de la cupidité. Ed. Les Liens qui Libèrent (LLL), Paris.

28. Stiglitz, J. E. (2010b). Le rapport Stiglitz : Pour une vraie réforme $d u$ système monétaire et financier international. Ed. Les Liens qui Libèrent (LLL), Paris.

29. Tsafack Nanfosso, R. \& Nguena, C. L. (2015). Importance des Politiques Financières dans la Croissance Economique en zone CEMAC: Approche en Données de Panel. African Development Review, 27(1), 52-66. 
30. Yao J-M., (2005). Approche Econométrique des Déterminants de la Rentabilité des Banques Européennes. MPRA Paper No. 17368, [En ligne] http://mpra.ub.uni-muenchen.de/17368/. 